\title{
miR-21-5p Suppresses Mitophagy to Alleviate Hyperoxia-Induced Acute Lung Injury by Directly Targeting PGAM5
}

\author{
Guoyue Liu $\mathbb{D}$, ${ }^{1}$ Mingjiang Qian $\mathbb{D}^{1},{ }^{1}$ Miao Chen $\mathbb{D}^{2},{ }^{2}$ Tao Chen $\mathbb{D}^{2},{ }^{2}$ and Song Qin $\mathbb{D}^{2}$ \\ ${ }^{1}$ The Second Affiliated Hospital of Zunyi Medical University, Zunyi, Guizhou 563000, China \\ ${ }^{2}$ Department of Critical Care Medicine, Affiliated Hospital of Zunyi Medical University, Zunyi, Guizhou 563000, China \\ Correspondence should be addressed to Miao Chen; chenmiao64@163.com
}

Received 17 June 2020; Accepted 5 August 2020; Published 20 November 2020

Academic Editor: Ali I. Abdalla

Copyright (c) 2020 Guoyue Liu et al. This is an open access article distributed under the Creative Commons Attribution License, which permits unrestricted use, distribution, and reproduction in any medium, provided the original work is properly cited.

Hyperoxia-induced acute lung injury (HALI) is a severe side effect of refractory hypoxemia treatment, for which no effective therapeutic strategy is available. Here, we found that the lung miR-21-5p level was significantly decreased in the rats subjected to hyperoxia. Further, we presented evidence that miR-21-5p was a crucial regulator of mitophagy and mitochondrial dysfunction. Moreover, it proved that miR-21-5p regulated hyperoxia-induced mitophagy and mitochondrial dysfunction by directly binding to the target gene PGAM5. In conclusion, for the first time, we found that miR-21-5p could directly suppress mitophagy and mitochondrial damage during HALI formation.

\section{Introduction}

Hyperoxia-induced acute lung injury (HALI) is a major clinical problem with high morbidity and mortality, which widely exists during the treatment of refractory hypoxemia [1]. It is reported that hyperoxia exposure stimulates the activation of inflammatory cells and thus results in pulmonary alveoli injury, alveolar-capillary leak, and the development of hyaline membranes [2,3]. Unfortunately, no effective therapeutic strategies are available for HALI treatment. Therefore, it is necessary to understand further the mechanism of HALI to investigate novel pharmacotherapy.

Accumulated evidence found that mitochondrial dysfunction plays a central role in HALI $[4,5]$. This dysfunction leads to the downregulation of ATP production, loss of mitochondrial membrane potential $(\Delta \Psi \mathrm{m})$, and reactive oxygen species (ROS) accumulation in endothelial or epithelial cells. Ultimately, it contributes to mitochondria-mediated cell death, such as apoptosis, autophagy, and necrocytosis [6]. At present, much attention has been given to the events that HALI-induced mitochondrial dysfunction could trigger type II alveolar epithelial cell (AEC-II) apoptosis or necrocytosis [7-9]. However, in the context of HALI, few studies focus on the selective autophagy of mitochondria, termed as mito- phagy. These findings result in a renewed interest in mitophagy and its role in HALI.

Nowadays, microRNAs (miRNAs) have been recently proved to be a new mediator of such stimuli, including ROS, mitochondrial dysfunction, and inflammation $[10,11]$. It is a kind of small, endogenous, and noncoding RNA molecules with 21-25 nucleotides. They take part in various pathologic processes by directly targeting the essential genes' $3^{\prime}$ untranslated regions ( $3^{\prime}$ UTR). In particular, Syed et al. had found that miR-34a was upregulated in the AEC-II cells and developing lungs, which were subjected to hyperoxia exposure. It suppressed the downstream target gene (angiopoietin-1) to induce the HALI and pulmonary arterial hypertension phenotype [3]. Similarly, in Prajapati et al.'s work, miRNAs have been confirmed to regulate neuron apoptosis by targeting transcript encoding subunit of mitochondrial complexes [12]. Thus, miRNAs have been considered a novel candidate for HALI therapy. However, whether miRNAs control mitophagy during HALI formation remains unclear. Of interest is miR-21-5p, which has been identified as a key mediator inducing acute lung injury and fibrosis $[13,14]$. Intriguingly, our previous studies have reported that miR21-5p was dramatically downregulated in the rat subjected to hyperoxic condition and inhibited apoptosis of type II 
alveolar epithelial cells through the PTEN/Akt pathway [2, 15]. However, whether miR-21-5p regulates mitophagy during HALI remains elusive.

For this purpose, we established a rat model to investigate the potential mechanism of HALI and found miR21-5p dramatically decreased during this process, which might associate with HALI formation. Then, we investigated the function of miR-21-5p in regulating mitochondrial damage and mitophagy in our hyperoxia-induced model. Our data demonstrated that the miR-21-5p could significantly inhibit mitochondrial damage and mitophagy in AEC-II cells to protect HALI through directly binding to PGAM5 3 'UTR.

\section{Materials and Methods}

2.1. Hyperoxia-Induced Acute Lung Injury Model (HALI Model). Thirty male Sprague-Dawley rats (200-220 g) were obtained from the Model Animal Research Center of Nanjing University. The rats were given free access to standard rodent food and tap water. All animal experiments were approved by the Animal Care and Use Committee of Zunyi Medical University. According to our previous study [16], the rats were randomly divided into three groups. In order to establish the acute lung injury model, rats were exposed to high concentration $(\geq 95 \%)$ oxygen at a flow rate of around $5.0 \mathrm{~L} / \mathrm{min}$ for $24 \mathrm{~h}$ or $48 \mathrm{~h}$. The control group was maintained in the normoxic environment.

2.2. Histological Analysis. The rats were anesthetized after different treatments. Rat lungs were isolated and immersed in $4 \%$ paraformaldehyde for $24 \mathrm{~h}$ at $4^{\circ} \mathrm{C}$. Lung tissues were then embedded in paraffin and sliced into five-micrometer sections. After dewaxing and hydration, the sections were immersed in hematoxylin for $5 \mathrm{~min}$ and then treated with an eosin solution for $1 \mathrm{~min}$. Then, the sections were dried and mounted with neutral gel. Finally, the sections were observed by light microscopy.

2.3. Measurement of Cytokines/Chemokines in Bronchoalveolar Lavage (BAL). After different treatments, the rats were euthanized, and the tracheas were isolated immediately. Then, $5 \mathrm{~mL}$ phosphate buffer saline was infused and recollected gently through the trachea (BAL fluid). After centrifuging at $1200 \times$ $\mathrm{g}$ for $5 \mathrm{~min}$, the supernatants were used to measure the concentration of malondialdehyde (MDA, Solarbio, Beijing, China) and tumor necrosis factor (TNF- $\alpha$, Abcam, USA) by the commercial kits (Nanjing Jiancheng, China) according to the manufacturer's instruction, respectively [17].

2.4. Lung Wet/Dry Weight Ratio. When the rats were euthanized, the lung tissues were isolated from the heart, trachea, and main bronchi. Each lung was wiped dry and weighed and then incubated at $70^{\circ} \mathrm{C}$ until the weight was not changed. The dry weight was recorded. The ratio was calculated to measure lung edema and water content according to the previous studies $[15,18]$.

2.5. Western Blot Analysis. The lung tissues were isolated from the mice with different treatments. The cells were col- lected after normoxia or hyperoxia exposure. Total proteins were then extracted using a Total Protein Extraction Kit (Synthgene Biotech, Nanjing, China) following the manufacturer's protocol. The protein concentration was measured by a BCA Protein Assay Kit (Synthgene Biotech, Nanjing, China) following the manufacturer's protocol. Equal protein of each group ( $30 \mu \mathrm{g}$ per lane) was separated by $10 \%$ SDSPAGE and transferred onto polyvinylidene fluoride membranes. After blocking with 5\% nonfat milk solution for $1 \mathrm{~h}$ at room temperature, the membranes were incubated with primary antibodies against light chain 3 (LC3) (1:1000, Abcam, Shanghai, China), p62 (1:1000, Abcam, Shanghai, China), Beclin1 (1:1000, Abcam, Shanghai, China), phosphoglycerate mutase family member 5 (PGAM5, 1:1000, Abcam, Shanghai, China), TIM23 (1:1000, Abcam, Shanghai, China), TOM20 (1:1000, Abcam, Shanghai, China), PINK1 (1:1000, Abcam, Shanghai, China), Parkin (1:1000, Abcam, Shanghai, China), COX IV (1:10000, Abcam, Shanghai, China), and GAPDH (1:10000, Abcam, Shanghai, China) overnight at $4^{\circ} \mathrm{C}$. Then, membranes were incubated with HRP-conjugated secondary antibody (anti-mouse IgG or anti-rabbit IgG, 1:5000, Abcam, Shanghai, China) for $2 \mathrm{~h}$ at room temperature. The pictures were detected by a HiSignal $^{\mathrm{TM}}$ ECL WB Detection Kit (Synthgene Biotech, Nanjing, China) following the manufacturer's protocol and quantified with ImageJ software.

2.6. Cell Culture. The isolation of AEC-II cells was performed based on previous studies $[16,17,19]$. In brief, rat lungs were obtained and washed by PBS several times to remove the blood and leukocytes. The tissues were then cut into small pieces and digested by $0.25 \%$ trypsin for $25 \mathrm{~min}$ at $37^{\circ} \mathrm{C}$. After filtering and centrifugation at $1000 \times \mathrm{g}$ for $5 \mathrm{~min}$, the cell pellets were collected. Cells were then digested by collagenase for $15 \mathrm{~min}$ at $37^{\circ} \mathrm{C}$ followed by several washes. In order to remove the fibroblasts, cell pellets were placed on a Petri flask overnight for differential adherence. The purity and survival rates of the cells were determined to be $>90 \%$. For the cell experiments, $500 \mu \mathrm{M} \mathrm{H}_{2} \mathrm{O}_{2}$ was used to simulate the hyperoxic condition [20]. The control group was maintained in the normoxic condition.

2.7. RNA Extraction and Reverse Transcription-Quantitative Polymerase Chain Reaction (RT-qPCR). The lung tissues were isolated from the mice with different treatments. The cells were collected after normoxia or hyperoxia exposure. For the detection of miRNA expression, total RNA was extracted using a Total RNA Extraction Kit (Synthgene Biotech, Nanjing, China) following the manufacturer's protocol. RNA quantity and purity were measured by a NanoDrop spectrophotometer (Thermo Fisher, Wilmington, DE, USA). The cDNA was synthesized using a Strand cDNA Synthesis kit (Takara Biotech, Dalian, China) following the manufacturer's protocol. The protocol was showed as follows: $42^{\circ} \mathrm{C}$ for $60 \mathrm{~min}, 70^{\circ} \mathrm{C}$ for $15 \mathrm{~min}$, and chilling at $4^{\circ} \mathrm{C}$. qRTPCR was then performed using TaqMan ${ }^{\mathrm{TM}}$ Fast Advanced Master Mix (Thermo Fisher, Wilmington, DE, USA) with the following procedure: $95^{\circ} \mathrm{C}$ for $5 \mathrm{~min}, 95^{\circ} \mathrm{C}$ for $15 \mathrm{~s}$ and extension for $1 \mathrm{~min}$ at $60^{\circ} \mathrm{C}$ for 40 cycles, and $60^{\circ} \mathrm{C}$ for 
5 min. U6 and GAPDH were used as an internal control. The data were normalized by the U6 or GAPDH with the $2^{-\triangle \triangle C T}$ method [21]. The primers are the following: miR-21-5p: forward $5^{\prime}$-GTCAATAGCTTATCAGACTGA-3' and reverse $5^{\prime}$ -GTTGGCTCTGGTGCAGGGTCCGAGGTATTCGCA-3 ${ }^{\prime}$; U6: forward 5'-CTCGCTTCGGCAGCACACG-3' and reverse $5^{\prime}$-AACGCTTCACGAATTTGCGT-3'; PGAM5: forward $5^{\prime}$ -GTGCCACTGGATTAGGGCAA-3' and reverse $5^{\prime}$-GGGA CTTCTCTGACCAGGCT- $3^{\prime}$; and GAPDH: forward $5^{\prime}$ -CAGGACCTCACTCATTGCCC- $3^{\prime}$ and reverse $5^{\prime}$-GACG GACACATTGGGGGTAG-3'.

2.8. Measurement of Mitochondrial Membrane Potential. The mitochondrial membrane potential $(\Delta \psi \mathrm{m})$ was monitored using a J Mitochondrial membrane potential assay kit with JC-1 (Beyotime, Shanghai, China) according to the manufacturer's instructions. Briefly, after treatment, the AEC-II cells were washed twice with HBSS (Sigma) and incubated in the dark with JC-1 $\left(7.5 \mu \mathrm{M} ; 30\right.$ minutes at $\left.37^{\circ} \mathrm{C}\right)$. Then, the cells were washed with JC-1 wash buffer. Fluorescence images were captured by confocal microscopy [18].

2.9. Detection of Mitophagy. For the mitophagy assay, AEC-II cells were seeded onto glass coverslips and transfected with GFP-LC3 plasmid using the HiTrans ${ }^{\text {TM }}$ LipoPlus Reagent (Synthgene Biotech, Nanjing, China). After $48 \mathrm{~h}$, cells were subjected to hyperoxia exposure $\left(500 \mu \mathrm{M} \mathrm{H}_{2} \mathrm{O}_{2}\right)$ with different treatments. Cells were then washed twice with PBS and incubated with $100 \mathrm{nM}$ MitoTracker Red (Molecular Probes, $\mathrm{M} 7512$ ) at $37^{\circ} \mathrm{C}$ for 15 minutes. The cells were then fixed with $4 \%$ paraformaldehyde for $10 \mathrm{~min}$ at room temperature, and images were observed by a confocal microscope (Fluoview FV1000, Olympus). The experiments were repeated at least three times independently.

2.10. Cell Transfection. AEC-II cells were seeded on a 6-well plate and transfected with $500 \mathrm{nM}$ miR-21-5p mimic or mimic control (mimic-NC), $0.5 \mu \mathrm{g}$ PGAM5 overexpression plasmid (PGAM5 OE), or control plasmid using a Lipofectamine $^{\mathrm{TM}} 3000$ reagent (Thermo Fisher Scientific, Inc.) following the manufacturer's protocol. The miR-21-5p mimic and related control, PGAM5 overexpression plasmid (PGAM5 $\mathrm{OE}$ ), and control plasmid were purchased from Synthgene (Nanjing, China). After $48 \mathrm{~h}$, the cells were collected for further experiments. For one group, the cells were cotransfected with PGAM5 OE $(0.5 \mu \mathrm{g})$ and miR-21-5p mimics (100 pmol) using the same reagent.

2.11. Luciferase Reporter Assay. A fragment of PGAM5 $3^{\prime}$ UTR, including the miR-21-5p binding sites, was predicted via TargetScan 7.2 (http://www.targetscan.org/). The luciferase reporter plasmids of pMIR-PGAM5-3' UTR-WT and pMIR-PGAM5-3'UTR-MUT were purchased from Synthgene (Nanjing, China). $10^{6}$ AEC-II cells were cotransfected with the plasmids $(0.5 \mu \mathrm{g})$ and miR-21-5p mimics (100 pmol), as well as the mimic control using HiTrans ${ }^{\mathrm{TM}}$ LipoPlus Reagent (Synthgene Biotech, Nanjing, China). The luciferase intensity was measured after $48 \mathrm{~h}$ using the DualGlo Luciferase Assay System (Promega, Shanghai, China) following the manufacturer's protocol. The data were normalized by renilla signals. The experiments were repeated at least three times independently.

2.12. Statistical Analysis. All data in this study were shown as the mean \pm Standard Error of Mean (SEM). Student's $t$-test was performed to determine the significance between two groups, and one-way or two-way ANOVA with Bonferroni post hoc tests for multiple groups. $P$ value $<0.05$ was considered statistically significant.

\section{Results}

3.1. Hyperoxia Activated Mitophagy and Induced Mitochondrial Dysfunction during HALI In Vivo. According to our previous study, we successfully established a lung injury model through hyperoxia administration [15]. Rats exposed to high oxygen showed diffuse pathological changes, including alveolar congestion and inflammatory cell infiltration. Moreover, the degree of injury was aggravated in a timedependent manner (Figure 1(a)). A significant timedependent increase had shown in the injury characteristics, including the W/D ratio of lung tissues and TNF- $\alpha$ in the BALF. Compared with the control group, the level of MDA had increased to $1.32 \pm 0.13 \mathrm{nmol} / \mathrm{mL}$ after hyperoxia exposure for $24 \mathrm{~h}(P<0.05)$ and further reached $1.44 \pm 0.15$ $\mathrm{nmol} / \mathrm{mL}$ at $48 \mathrm{~h}$ after hyperoxia administration (Figure $1(\mathrm{~b})$ ).

To determine the effect of hyperoxia on mitophagy in vivo, we first assessed the changes in mitophagy protein markers using western blot. The result showed that the expression of mitophagy marker PINK1 and Parkin was significantly elevated along with the prolonged hyperoxia exposure. As a critical pathway in mitophagy regulation, our results indicated that the mitophagy occurred during HALI. Besides, hyperoxia exposure significantly regulated the expression of autophagy-related proteins, with an 8-fold increase of the LC3-II/LC3-I ratio and 5-fold upregulation of Beclin1 expression, as well as a significant decline of the p62 level, in the lung tissue of the hyperoxia $48 \mathrm{~h}$ group as compared to the control group. In addition, we found that the expression of mitophagy markers like TIM23 and TOM20 was dropped as compared to that in the control group, suggesting that the mitochondrial content was lost under the hyperoxic condition (Figures $1(\mathrm{c})$ and $1(\mathrm{~d})$ ). We then checked the mitochondrial function by a fluorescence assay. AEC-II cells were isolated from lung tissues of normal rats and treated in vitro with $\mathrm{H}_{2} \mathrm{O}_{2}$ exposure for $24 \mathrm{~h}$ or $48 \mathrm{~h}$. As shown in Figure 2(a), compared with the control group, the red signal of J-aggregate was time-dependently reduced, while the green signal of monomer was inclined in the $\mathrm{H}_{2} \mathrm{O}_{2}$-treated groups.

Next, we sought to identify whether there was a link between severe mitochondrial damage and the formation of the autophagosome in AEC-II cells. Cells were transfected with both MitoTracker Red and GFP-LC3, following exposure to $\mathrm{H}_{2} \mathrm{O}_{2}$ for $24 \mathrm{~h}$ or $48 \mathrm{~h}$. As shown in Figure 2(b), with time increased, the presence of LC3 puncta was raised and showed a significant overlap with mitochondria. All of these data showed that mitochondrial damage has been aggravated 

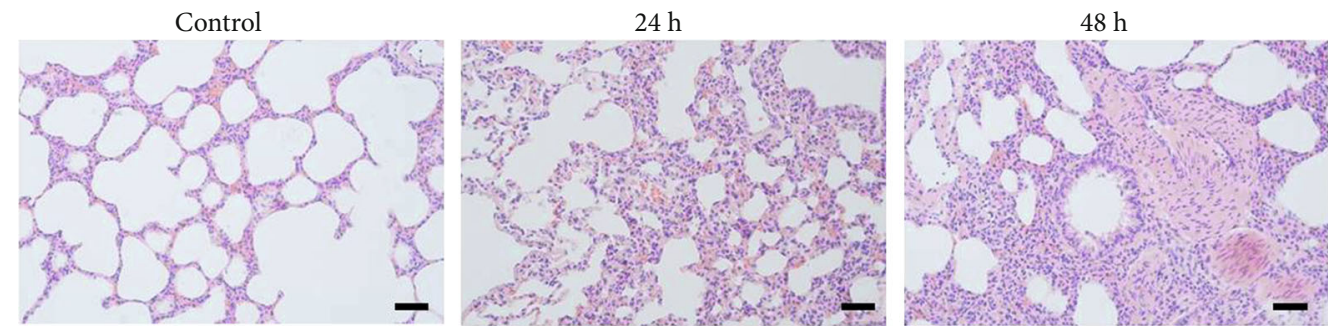

(a)
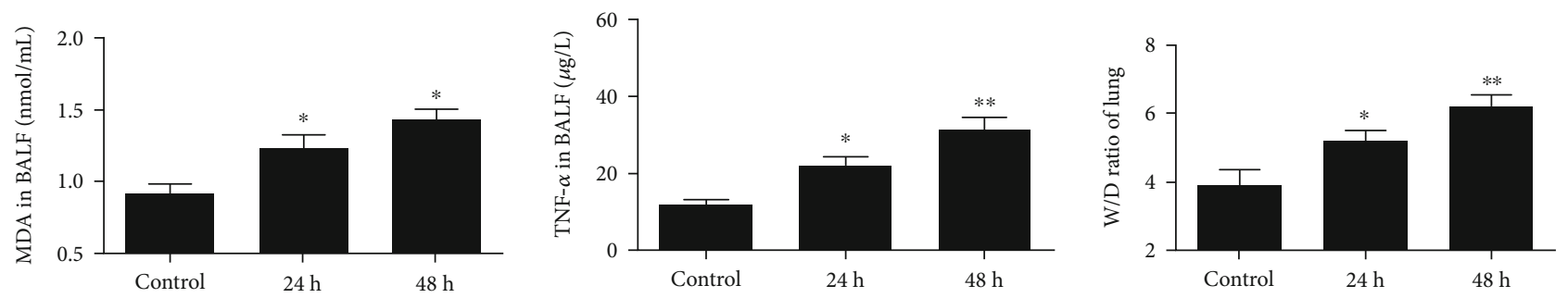

(b)
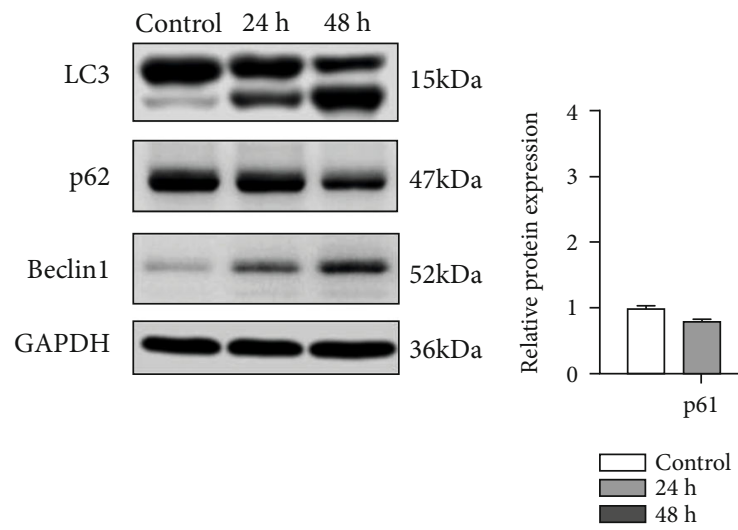

(c)

(d)

Figure 1: Hyperoxia exposure induced mitophagy during HALI formation. (a) Lung tissues of rats were collected and subjected to hematoxylin and eosin staining. The images were observed under light microscopy (magnification, $\times 200$ ). Images showed representative examples of three independent experiments. Bar $=20 \mu \mathrm{m}$. (b) After different treatments, the W/D ratio of the lung tissues was detected; serum TNF- $\alpha$ and MDA levels were determined using ELISA. (c) Representative blots and densitometric analysis were shown for the expression level of LC3, PINK1, Parkin, and COX IV in rat lung tissues. (d) Representative blots and densitometric analysis were shown for the expression level of TIM23, TOM20, p62, Beclin1, and GAPDH in rat lung tissues. $N=6$. Data were presented as the mean \pm SEM. ${ }^{*} P<0.05,{ }^{* *} P<0.01$, and ${ }^{* * *} P<0.001$.

under a long-time hyperoxia administration and thus initiated related mitophagy in the AEC-II cells.

\section{4. miR-21-5p Alleviated Mitochondrial Dysfunction and Decreased Mitophagy in AEC-II Cell Model}

Our previous study has demonstrated that hyperoxia exposure markedly decreased miR-21-5p expression in AEC-II cells. Here, we also confirmed that the expression of miR-21-5p was time-dependently downregulated in the rats subjected to hyperoxia. The level of miR-21-5p was dropped about 2-folds when the rats were maintained in the hyperoxia condition for $48 \mathrm{~h}(P<0.05)$. Consistently, we investigated that the miR-21$5 \mathrm{p}$ level was also decreased along with the prolongation of stim- ulation time in vitro. The most significant decrease in it was showed in the $48 \mathrm{~h}$ treatment group $(P<0.05)$ (Figure 3$)$.

To investigate the role of miR-21-5p in HALI, $\mathrm{H}_{2} \mathrm{O}_{2}$ treated AEC-II cells were transfected with miR-21-5p mimic or mimic-NC. As expected, compared with the control group, the mitochondrial membrane potential level was decreased markedly, while miR-21-5p mimic treatment significantly reversed the reduction of the mitochondrial membrane potential level to repair the mitochondrial dysfunction. Then, we used the fluorescence of mitochondria and lysosome to observe the mitophagy. The overlap of mitochondria and lysosome was induced, comparable to the cells exposed to hyperoxic condition alone (Figures 4(a) and 4(b)).

Accordingly, $\mathrm{H}_{2} \mathrm{O}_{2}$ administration increased the expression level of PINK1 and Parkin, while downregulating the protein level of TIM23 and TOM20. In addition, the LC3- 


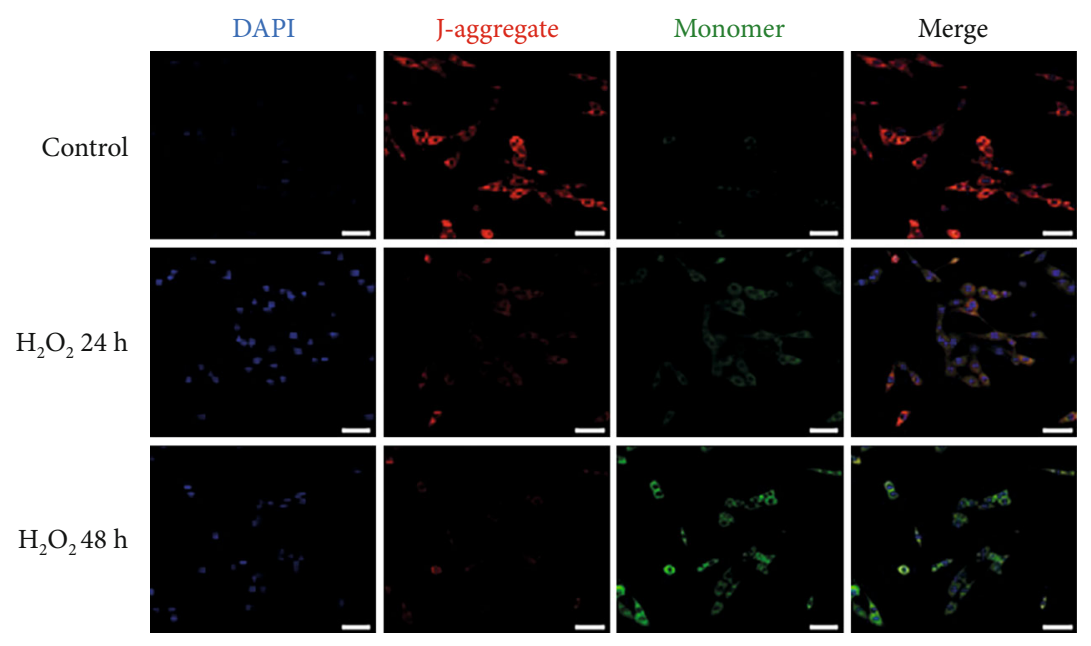

(a)

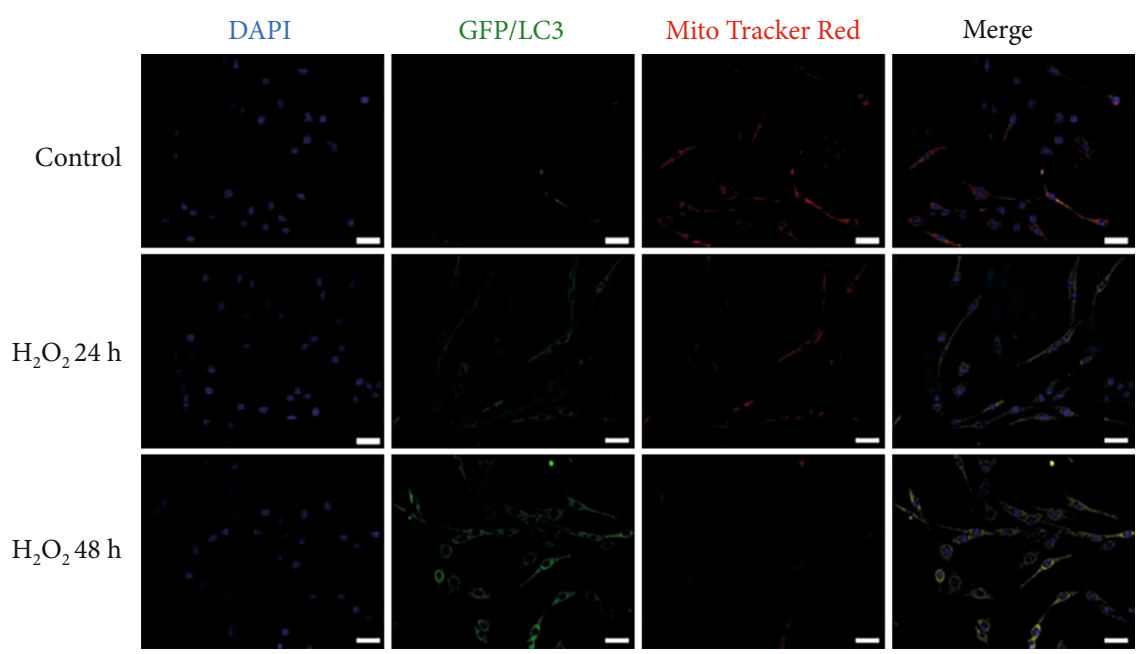

(b)

FIGURE 2: Hyperoxia exposure induced mitochondrial dysfunction during HALI formation. AEC-II cells were isolated from the rats and treated with $500 \mu \mathrm{M} \mathrm{H}_{2} \mathrm{O}_{2}$ for 24 or $48 \mathrm{~h}$. (a) Representative confocal microscopy images of JC-1 staining and (b) MitoTracker Red/GFP-LC3 staining of the cells with different treatments were performed. Images showed representative examples from three independent experiments. Bar $=20 \mu \mathrm{m}$.

II/LC3-I ratio was significantly increased under the $\mathrm{H}_{2} \mathrm{O}_{2}$ exposure than that in the normoxia+mimic-NC group, which was $39.3 \pm 5.32$ and $3.22 \pm 1.21$, respectively $(P<0.001)$. Similarly, a 40 -fold upregulation of the Beclin1 expression had been observed in the $\mathrm{H}_{2} \mathrm{O}_{2}+$ mimic-NC group $(P<0.001)$. Contrarily, compared with the normoxia + mimic-NC group, there was a marked decline in the p62 expression level of the $\mathrm{H}_{2} \mathrm{O}_{2}+$ mimic-NC group $(P<0.01)$. However, the anomalous change of these protein markers had been reversed after miR-21-5p mimic administration (Figure 4(c)). These data offered evidence that miR-21-5p played an important role in mitophagy and mitochondrial function during hyperoxia treatment.

\section{5. miR-21-5p Directly Targets PGAM5 in AEC-II Cells}

To identify the target of miR-21-5p, the TargetScan database (http://www.targetscan.org) was used to search the potential targets. Among them, PGAM5 was the candidate gene with

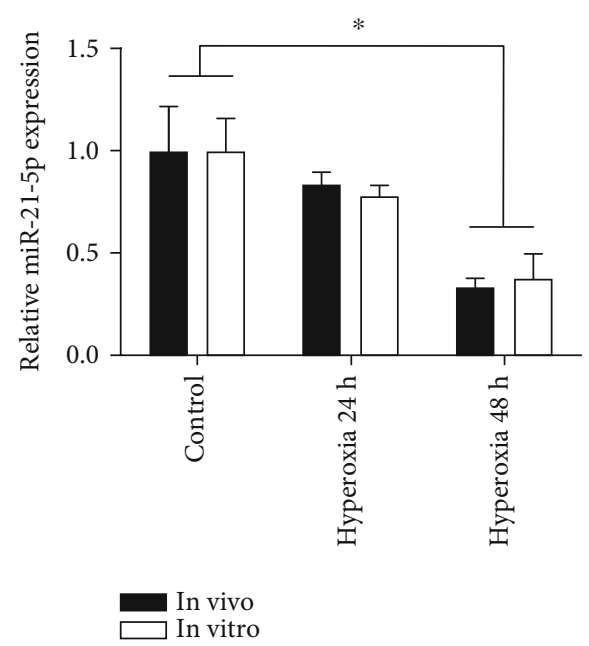

Figure 3: The expression level of miR-21-5p in hyperoxic rat lung tissues and AEC-II cells with different treatments. Data are presented as the mean \pm SEM. ${ }^{*} P<0.05 v s$. the control group. 


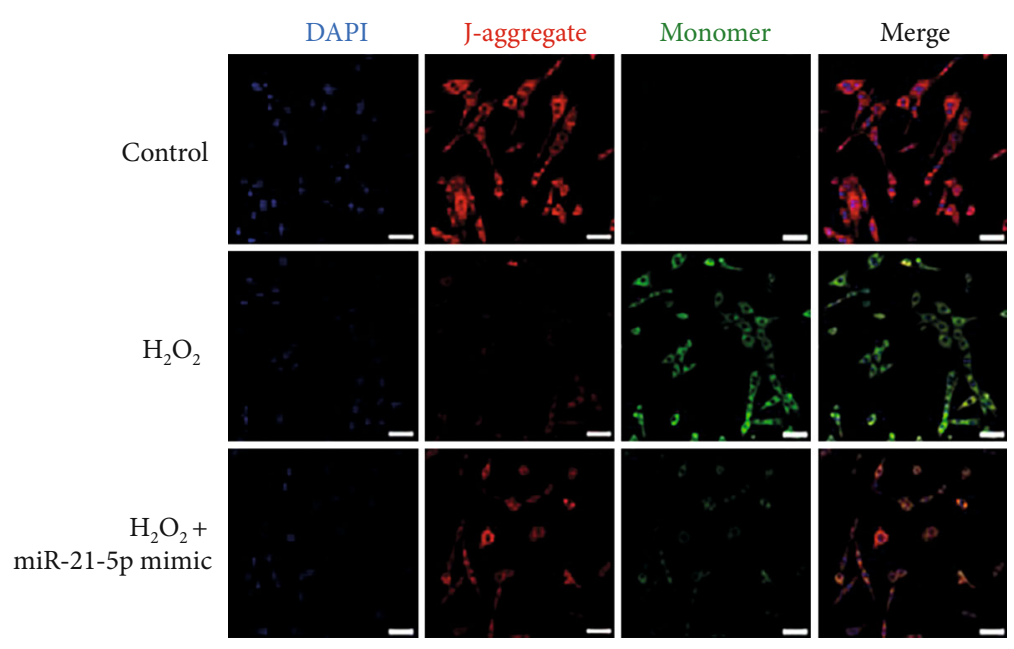

(a)

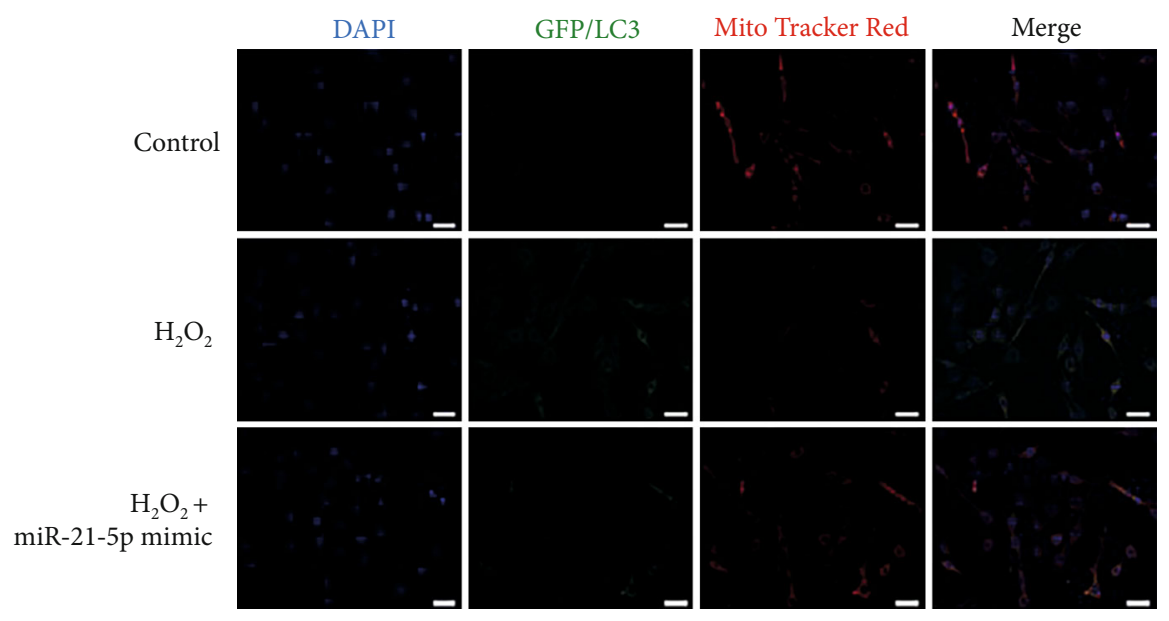

(b)

FIgUre 4: Continued. 

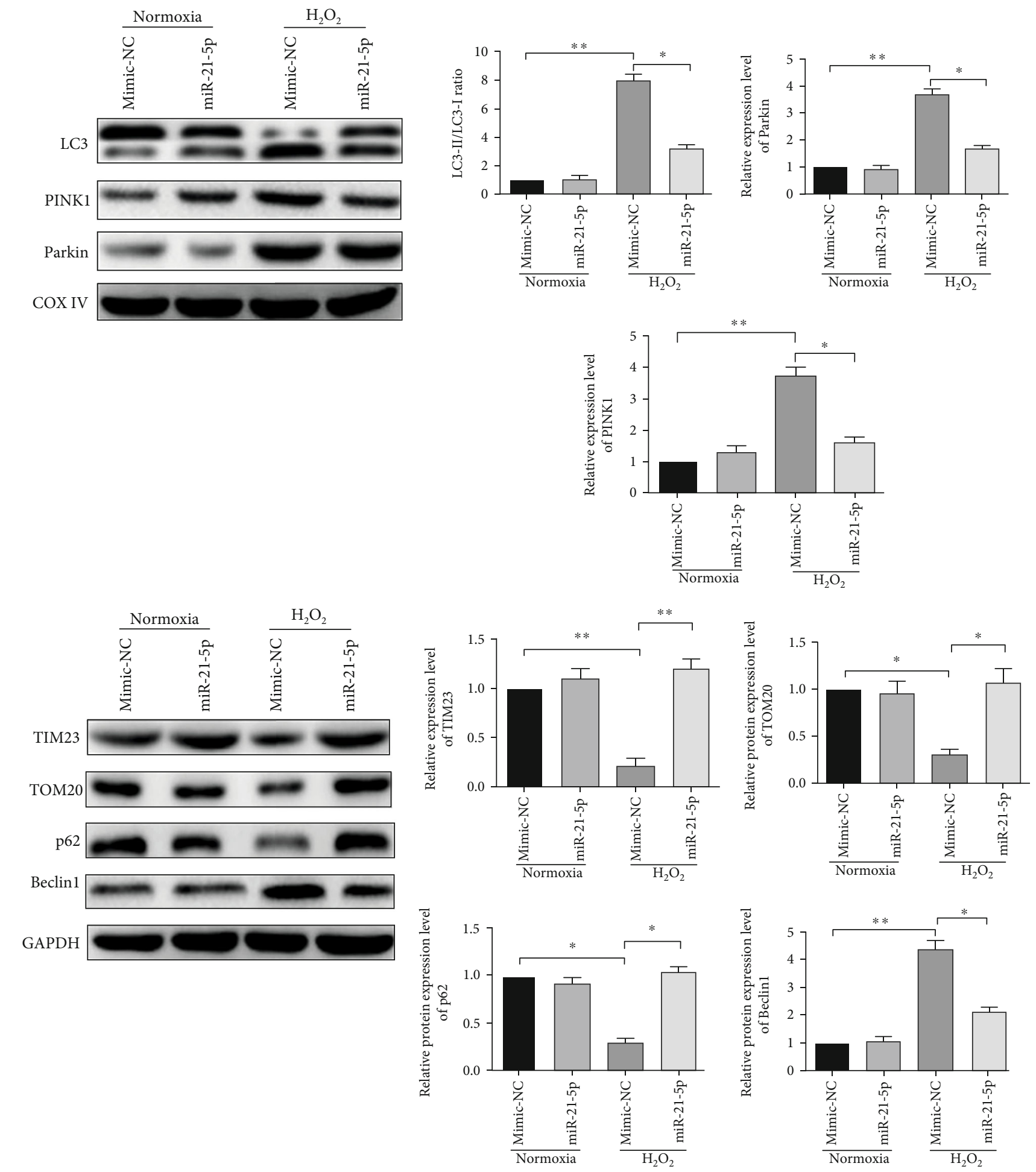

(c)

FIGURE 4: Effect of miR-21-5p on mitochondrial damage and mitophagy of AEC-II cells. (a) Representative confocal microscopy images of JC-1 staining and (b) MitoTracker Red/GFP-LC3 staining of the AEC-II cells with different treatments were performed. Images showed representative examples from three independent experiments. Bar $=20 \mu \mathrm{m}$. (c) Representative blots and densitometric analyses were shown for the expression level of LC3, PINK1, Parkin, and COX IV in AEC-II cells after different treatments. (d) Representative blots and densitometric analyses were shown for the expression level of TIM23, TOM20, p62, Beclin1, and GAPDH in AEC-II cells with different treatments. $N=6$. Data are presented as the mean \pm SEM. ${ }^{*} P<0.05,{ }^{* *} P<0.01$, and ${ }^{* * *} P<0.001$. 

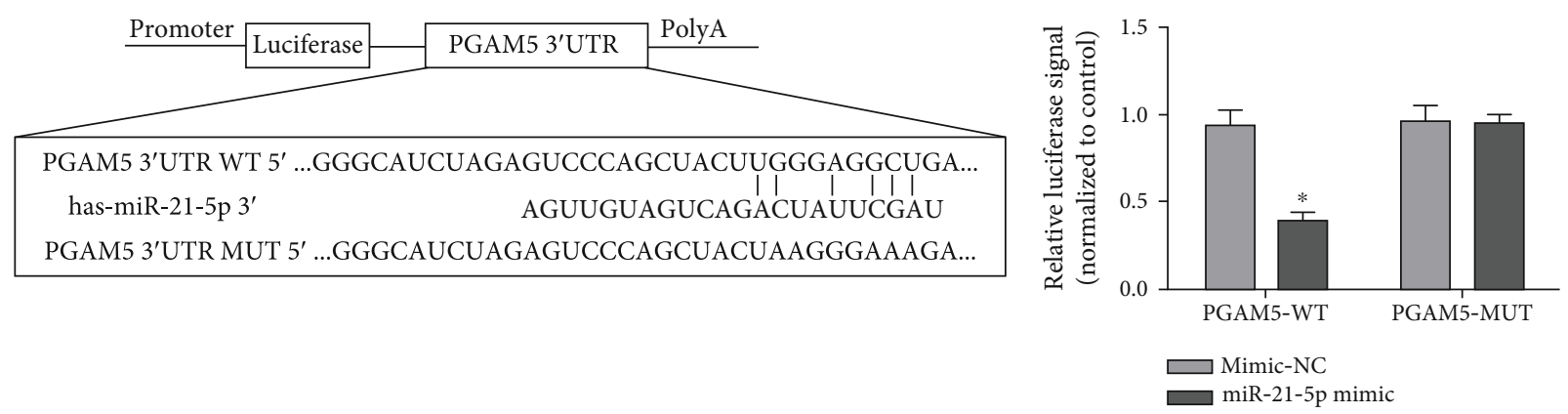

(a)

(b)
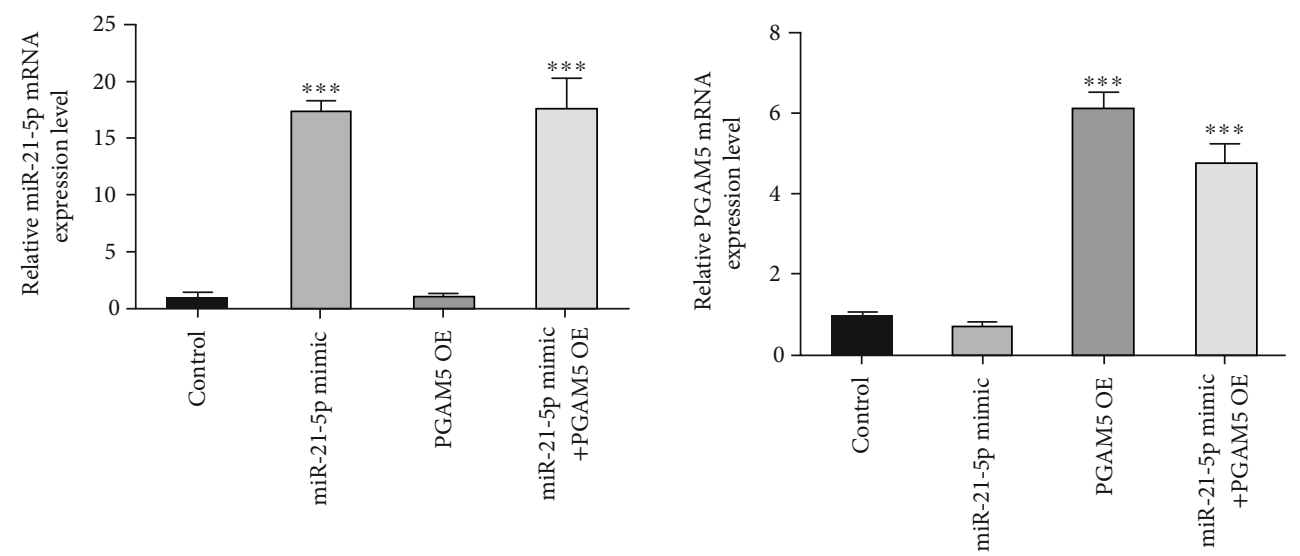

(c)

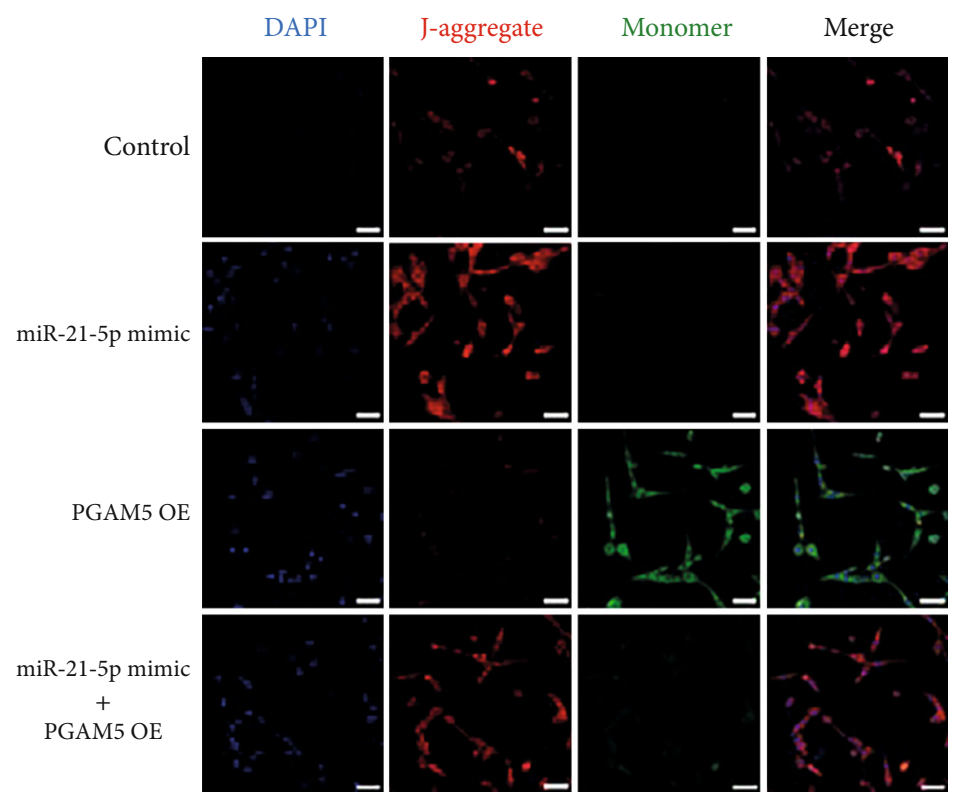

(d)

Figure 5: Continued. 


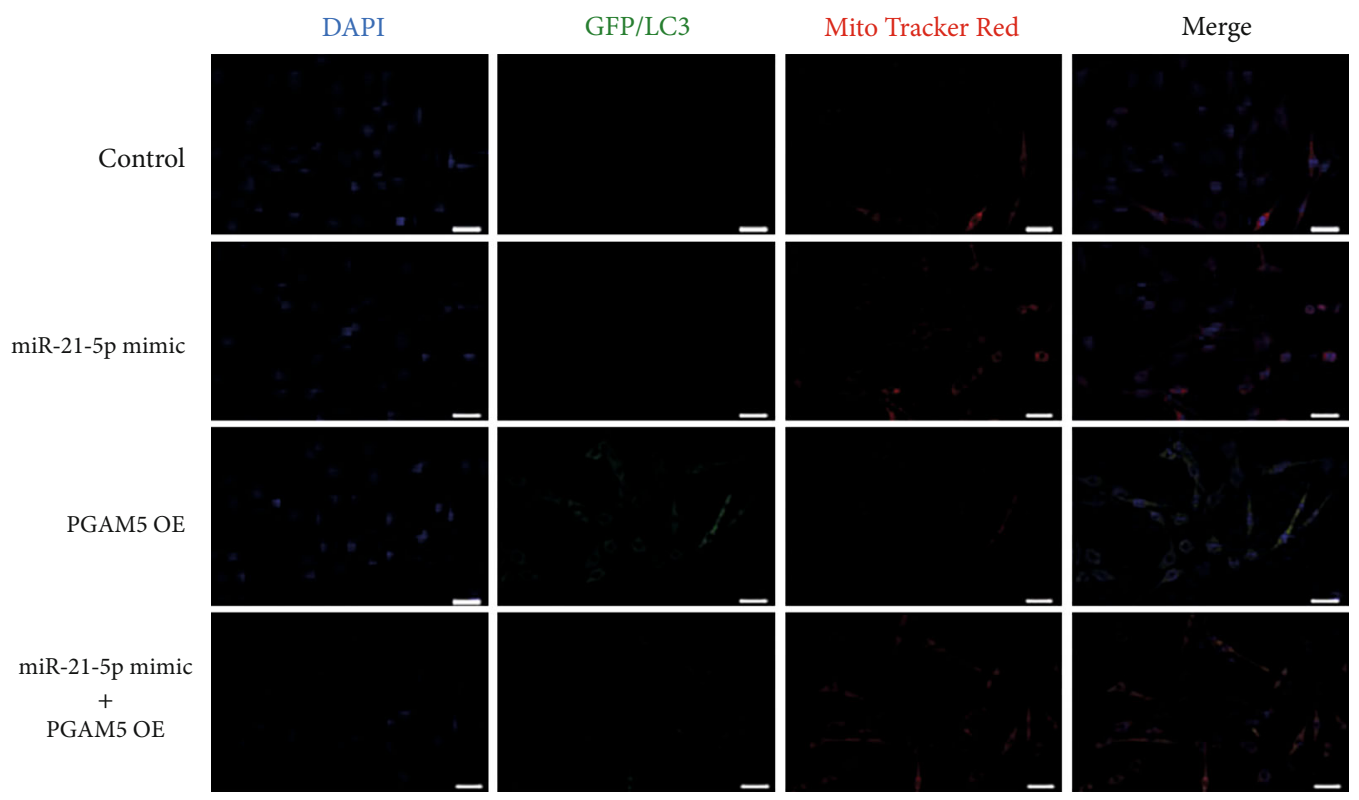

(e)
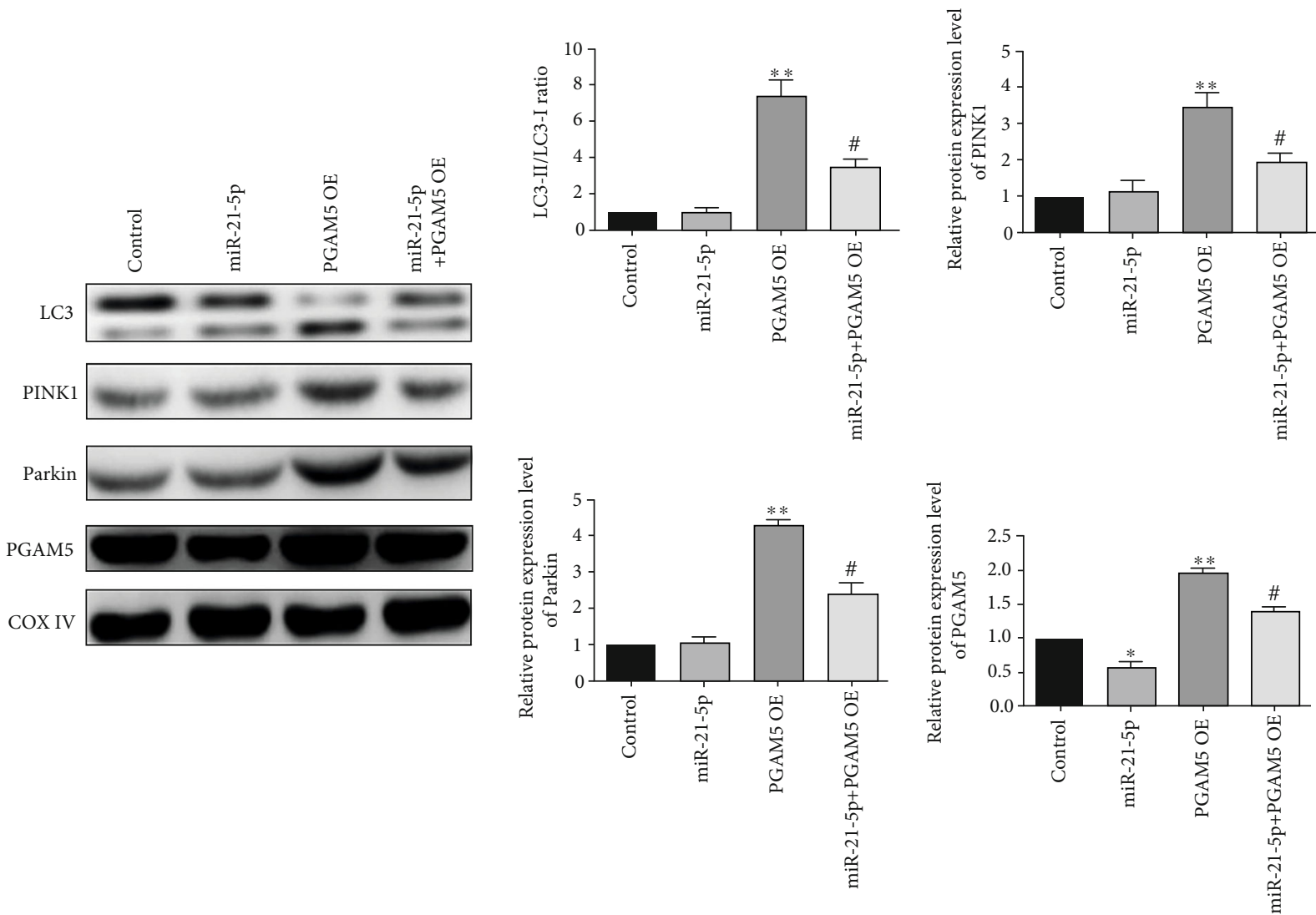

(f)

FIGURE 5: miR-21-5p directly targets PGAM5 to regulate mitophagy in AEC-II cells. (a) Sequence alignment of the PGAM5 $3{ }^{\prime}$ UTR with miR21-5p. (b) The activity of luciferase reporters containing wild-type (WT) or mutant (MUT) PGAM5 $3^{\prime} \mathrm{UTR}$ that were treated with miR-21-5p mimics or mimic control (mimic-NC). (c) The transfection efficiency of miR-21-5p mimic and PGAM5 plasmid in AEC-II cells. (d) Representative confocal microscopy images of JC-1 staining and (e) MitoTracker Red/GFP-LC3 staining of the AEC-II cells with different treatments were performed. Images showed representative examples from three independent experiments. Bar $=20 \mu \mathrm{m}$. (f) Representative blots and densitometric analyses were shown for the expression of PGAM5, LC3, PINK1, Parkin, and COX IV in AEC-II cells after different treatments. $N=6$. Data are presented as the mean \pm SEM. ${ }^{*} P<0.05,{ }^{* *} P<0.01$, and ${ }^{* * *} P<0.001 v s$. the control group; ${ }^{\#} P<0.05$, ${ }^{\# \#} P<0.01$, and ${ }^{\# \# \#} P<0.001$ vs. the PGAM5 OE group. 
the highest score. The binding sites between miR-21-5p and PGAM5 are presented in Figure 5(a). In order to determine the mechanism of miR-21-5p, the wild-type or mutant PGAM5 $3^{\prime}$ UTR were designed and constructed in the downstream of a luciferase reporter gene. The luciferase intensity was reduced obviously by a miR-21-5p mimic in the wild-type group rather than the mutant one $(P<0.05)$, indicating that miR-21-5p specially banded to the $3^{\prime}$ UTR of PGAM5-WT and regulated the PGAM5 expression negatively (Figure 5(b)).

\section{Hyperoxia Activated Mitophagy and Induced Mitochondrial Dysfunction via miR-21-5p/PGAM5 Axis}

To further confirm the function of the miR-21-5p/PGAM5 axis, we transferred PGAM5 plasmid into AEC-II cells under normoxic condition and treated them using miR-21-5p mimic. As shown in Figure 5(c), a 16-fold increase and 6fold upregulation were observed in the cells after miR-21$5 \mathrm{p}$ mimic or PGAM5 plasmid transfection, respectively. Besides, the overexpression of PGAM5 could highly decline the mitochondrial membrane potential level and increase the expression level of LC3 in mitochondria. With miR-21$5 \mathrm{p}$ treatment, these phenomena had been reversed (Figures 5(d) and 5(e)). Consistently, as shown in Figure 5(f), miR-21-5p treatment notably decreased the protein level of PGAM5 as compared to the control group $(P<0.05)$. Moreover, the western blot results demonstrated that the overexpression of PGAM5 in the cells might dramatically upregulate the LC3-II/LC-I ratio and the expression level of PINK1 and Parkin $(P<0.01)$. However, when the cells were transfected with miR-21-5p mimic and PGAM5 plasmid, the protein levels of these markers were decreased, which suggested that the phenomena of mitophagy were abolished. These experiments revealed that miR-21-5p might suppress mitophagy through binding to PGAM5 $3{ }^{\prime}$ UTR.

\section{Discussion}

Exposure to the high concentrations of oxygen causes a massive increase in reactive oxygen metabolites, leading to severe inflammation and cell apoptosis in the lungs, which limits the application of oxygen [22]. In this study, we first successfully established a rat model of HALI. After incubation with a high level of oxygen, the rats showed significant lung injury in a time-dependent manner. We further found that the expression level of LC3, p62, and Beclin1 was regulated by hyperoxia exposure, indicating that autophagy had been activated during HALI. Previous studies showed that autophagy is an evolutionarily conserved physiological process, which upregulated rapidly under various stress conditions, including oxidative stress and hyperoxia [23, 24].

As a kind of special autophagy, mitophagy acts as a selective scavenger of mitochondria in physiological and pathological conditions. Numerous studies have proved that the PINK1-Parkin pathway might be activated during the process of mitophagy, accompanied by increased LC3 matura- tion and declined expression of mitophagy markers such as TIM23 and TOM20, which serve as the biomarker for mitophagy identification $[25,26]$. Nowadays, emerging evidence shows that mitophagy may subsequently attenuate apoptosis to maintain normal tissue homeostasis [27]. However, in some specific cellular settings, mitophagy may lead to cell death [28, 29]. In this work, after $\mathrm{H}_{2} \mathrm{O}_{2}$ treatment, a time-dependent mitochondrial dysfunction had been performed with the loss of the mitochondrial membrane potential level. Consistently, an increasing number of mitochondria surrounded by lysosomes have been detected with $24 \mathrm{~h}$ and $48 \mathrm{~h}$ treatment, suggesting mitochondrial mass loss and mitochondrial dysfunction occurrence during HALI. Our findings also demonstrated that $\mathrm{H}_{2} \mathrm{O}_{2}$ exposure could activate mitophagy as evidenced by the increased LC3-II/LC3-I ratio and activated PINK-Parkin pathway in AEC-II cells.

MicroRNAs (miRNAs) can negatively regulate gene expression at the posttranscriptional level [30]. Many researchers have demonstrated that overexpression of miR21-5p in some types of cancer cells has been proved to involve in cellular proliferation and apoptosis [31]. For example, previous studies reported that miR-21-5p inhibits apoptosis in a breast cancer mouse model and gemcitabineinduced cells $[32,33]$. Interestingly, in this work, we found that miR-21-5p was expressed both in rat lungs and AEC-II cells. The level of it was significantly decreased after longtime hyperoxia treatment. Then, using genetic gain-offunction strategies, we revealed a promising causal role of increased miR-21-5p, protecting against mitochondrial damage and related mitophagy in AEC-II cells.

To further confirm the target of miR-21-5p, the TargetScan database (http://www.targetscan.org) was used. We found that PGAM5 was a possible target of miR-21-5p. As a critical driver of mitochondrial dysfunction, PGAM5 played an essential role in the intrinsic apoptosis and necrosis pathway [34,35]. In our model, we identified that overexpression of PGAM5 could highly reduce the mitochondrial membrane potential level and promote mitophagy. miR-21-5p negatively regulated expression of PGAM5 through directly banding the $3^{\prime}$ UTR of PGAM5 mRNA. Our data thus revealed the role of miR-21$5 p$ and PGAM5, which was believed to be necessary for the regulation of mitochondrial function and the related HALI. All these results indicated that miR-21-5p inhibited hyperoxiainduced HALI, through directly suppressing PGAM5 function in AEC-II cells.

In summary, this study identified that mitophagy was involved in the pathological process of hyperoxia-induced acute lung injury. miR-21-5p contributed to protecting HALI by influencing mitophagy through regulation of the PGAM5 gene.

\section{Data Availability}

The data used to support the findings of this study are available from the corresponding author upon request.

\section{Conflicts of Interest}

The authors declare that they have no conflict of interest. 


\section{Authors' Contributions}

Guoyue Liu and Mingjiang Qian contributed equally to this work. Mingjiang Qian is the co-first author.

\section{Acknowledgments}

The authors gratefully acknowledge the financial support from the National Natural Sciences Foundation of China (81560019) and the science and technology program of Guizhou Province, China (NO. [2017]7115).

\section{References}

[1] M. F. Nyp, S. M. Mabry, A. Navarro et al., "Lung epithelialspecific TRIP-1 overexpression maintains epithelial integrity during hyperoxia exposure," Physiological Reports, vol. 6, no. 5, p. e13585, 2018.

[2] S. Qin, M. Chen, H. Ji et al., "miR-21-5p regulates type II alveolar epithelial cell apoptosis in hyperoxic acute lung injury," Molecular Medicine Reports, vol. 17, no. 4, pp. 5796-5804, 2018.

[3] M. Syed, P. Das, A. Pawar et al., "Hyperoxia causes miR-34amediated injury via angiopoietin-1 in neonatal lungs," Nature Communications, vol. 8, no. 1, p. 1173, 2017.

[4] V. R. Narala, J. Fukumoto, H. Hernandez-Cuervo et al., "Akap1 genetic deletion increases the severity of hyperoxiainduced acute lung injury in mice," American Journal of Physiology. Lung Cellular and Molecular Physiology, vol. 314, no. 5, pp. L860-L870, 2018.

[5] S. S. Patil, H. Hernandez-Cuervo, J. Fukumoto et al., "Alda-1 attenuates hyperoxia-induced mitochondrial dysfunction in lung vascular endothelial cells," Aging, vol. 11, no. 12, pp. 3909-3918, 2019.

[6] G. C. Higgins and M. T. Coughlan, "Mitochondrial dysfunction and mitophagy: the beginning and end to diabetic nephropathy?"” British Journal of Pharmacology, vol. 171, no. 8, pp. 1917-1942, 2014.

[7] L. Li, W. Wu, W. Huang, G. Hu, W. Yuan, and W. Li, "NF- $\kappa$ B RNAi decreases the $\mathrm{Bax} / \mathrm{Bcl}-2$ ratio and inhibits TNF- $\alpha$ induced apoptosis in human alveolar epithelial cells," Inflammation Research, vol. 62, no. 4, pp. 387-397, 2013.

[8] V. Panduri, S. A. Weitzman, N. Chandel, and D. W. Kamp, "The mitochondria-regulated death pathway mediates asbestos-induced alveolar epithelial cell apoptosis," American Journal of Respiratory Cell and Molecular Biology, vol. 28, no. 2, pp. 241-248, 2003.

[9] M. Perl, C. S. Chung, U. Perl et al., "Fas-induced pulmonary apoptosis and inflammation during indirect acute lung injury," American Journal of Respiratory and Critical Care Medicine, vol. 176, no. 6, pp. 591-601, 2007.

[10] R. Rao, P. Nagarkatti, and M. Nagarkatti, "Role of miRNA in the regulation of inflammatory genes in staphylococcal enterotoxin B-induced acute inflammatory lung injury and mortality," Toxicological Sciences, vol. 144, no. 2, pp. 284-297, 2015.

[11] M. Murri and H. El Azzouzi, "MicroRNAs as regulators of mitochondrial dysfunction and obesity," American Journal of Physiology. Heart and Circulatory Physiology, vol. 315, no. 2, pp. H291-H302, 2018.

[12] P. Prajapati, L. Sripada, K. Singh, K. Bhatelia, R. Singh, and R. Singh, "TNF- $\alpha$ regulates miRNA targeting mitochondrial
complex-I and induces cell death in dopaminergic cells," Biochimica et Biophysica Acta, vol. 1852, no. 3, pp. 451-461, 2015.

[13] G. Liu, A. Friggeri, Y. Yang et al., "miR-21 mediates fibrogenic activation of pulmonary fibroblasts and lung fibrosis," Journal of Experimental Medicine, vol. 207, no. 8, pp. 1589-1597, 2010.

[14] J. W. Li, L. Wei, Z. Han, and Z. Chen, "Mesenchymal stromal cells-derived exosomes alleviate ischemia/reperfusion injury in mouse lung by transporting anti-apoptotic miR-21-5p," European Journal of Pharmacology, vol. 852, pp. 68-76, 2019.

[15] S. Qin, H. Wang, G. Liu, H. Mei, and M. Chen, "miR-21-5p ameliorates hyperoxic acute lung injury and decreases apoptosis of AEC II cells via PTEN/AKT signaling in rats," Molecular Medicine Reports, vol. 20, no. 6, pp. 4953-4962, 2019.

[16] G. Liu, H. Mei, M. Chen et al., "Protective effect of agmatine against hyperoxia-induced acute lung injury via regulating lncRNA gadd7," Biochemical and Biophysical Research Communications, vol. 516, no. 1, pp. 68-74, 2019.

[17] M. N. Ballinger, M. W. Newstead, X. Zeng et al., "TLR signaling prevents hyperoxia-induced lung injury by protecting the alveolar epithelium from oxidant-mediated death," Journal of Immunology, vol. 189, no. 1, pp. 356-364, 2012.

[18] Q. Zhang, D. Wu, Y. Yang, T. Liu, and H. Liu, "Dexmedetomidine alleviates hyperoxia-induced acute lung injury via inhibiting NLRP3 inflammasome activation," Cellular Physiology and Biochemistry, vol. 42, no. 5, pp. 1907-1919, 2017.

[19] J. Lin, J. Tian, L. Wang et al., “Apoptosis and surfactant protein-C expression inhibition induced by lipopolysaccharide in AEC II cell may associate with NF- $\kappa \mathrm{B}$ pathway," The Journal of Toxicological Sciences, vol. 42, no. 1, pp. 53-61, 2017.

[20] Y. Wang, S. I. Feinstein, and A. B. Fisher, "Peroxiredoxin 6 as an antioxidant enzyme: protection of lung alveolar epithelial type II cells from H2O2-induced oxidative stress," Journal of Cellular Biochemistry, vol. 104, no. 4, pp. 1274-1285, 2008.

[21] K. J. Livak and T. D. Schmittgen, "Analysis of relative gene expression data using real-time quantitative PCR and the $2^{-\triangle \Delta \mathrm{CT}}$ method," Methods, vol. 25, no. 4, pp. 402-408, 2001.

[22] C. R. Carvalho, G. de Paula Pinto Schettino, B. Maranhao, and E. P. Bethlem, "Hyperoxia and lung disease," Current Opinion in Pulmonary Medicine, vol. 4, no. 5, pp. 300-304, 1998.

[23] K. Nakahira, M. A. P. Porras, and A. M. K. Choi, "Autophagy in pulmonary diseases," American Journal of Respiratory and Critical Care Medicine, vol. 194, no. 10, pp. 1196-1207, 2016.

[24] S. W. Ryter, K. Nakahira, J. A. Haspel, and A. M. K. Choi, "Autophagy in pulmonary diseases," Annual Review of Physiology, vol. 74, no. 1, pp. 377-401, 2012.

[25] Q. Jin, R. Li, N. Hu et al., "DUSP1 alleviates cardiac ische$\mathrm{mia} /$ reperfusion injury by suppressing the Mff-required mitochondrial fission and Bnip3-related mitophagy via the JNK pathways," Redox Biology, vol. 14, pp. 576-587, 2018.

[26] G. Ashrafi and T. L. Schwarz, "The pathways of mitophagy for quality control and clearance of mitochondria," Cell Death and Differentiation, vol. 20, no. 1, pp. 31-42, 2013.

[27] H. Dai, Y. Deng, J. Zhang et al., "PINK1/Parkin-mediated mitophagy alleviates chlorpyrifos-induced apoptosis in $\mathrm{SH}$ SY5Y cells," Toxicology, vol. 334, pp. 72-80, 2015.

[28] A. Eisenberg-Lerner, S. Bialik, H. U. Simon, and A. Kimchi, "Life and death partners: apoptosis, autophagy and the crosstalk between them," Cell Death and Differentiation, vol. 16, no. 7, pp. 966-975, 2009.

[29] Q.-p. Liu, D.-x. Zhou, L. Sun et al., "Bone marrow mesenchymal stem cells ameliorates seawater-exposure-induced acute 
lung injury by inhibiting autophagy in lung tissue," Pathology Research International, vol. 2014, Article ID 104962, 7 pages, 2014.

[30] L. He and G. J. Hannon, "MicroRNAs: small RNAs with a big role in gene regulation," Nature Reviews. Genetics, vol. 5, no. 7, pp. 522-531, 2004.

[31] L. B. Frankel, N. R. Christoffersen, A. Jacobsen, M. Lindow, A. Krogh, and A. H. Lund, "Programmed cell death 4 (PDCD4) is an important functional target of the microRNA miR-21 in breast cancer cells," The Journal of Biological Chemistry, vol. 283, no. 2, pp. 1026-1033, 2008.

[32] M. L. Si, S. Zhu, H. Wu, Z. Lu, F. Wu, and Y. Y. Mo, "miR-21mediated tumor growth," Oncogene, vol. 26, no. 19, pp. 27992803, 2007.

[33] F. Meng, R. Henson, M. Lang et al., "Involvement of human micro-RNA in growth and response to chemotherapy in human cholangiocarcinoma cell lines," Gastroenterology, vol. 130, no. 7, pp. 2113-2129, 2006.

[34] W. Xu, L. Jing, Q. Wang et al., "Bax-PGAM5L-Drp1 complex is required for intrinsic apoptosis execution," Oncotarget, vol. 6, no. 30, pp. 30017-30034, 2015.

[35] Z. Wang, H. Jiang, S. Chen, F. Du, and X. Wang, "The mitochondrial phosphatase PGAM5 functions at the convergence point of multiple necrotic death pathways," Cell, vol. 148, no. 1-2, pp. 228-243, 2012. 